Revue d'histoire de l'Amérique française

Q8. REVUE D.HISTOIRE DE L'AMÉRIQUE FRANÇAISE

\title{
L’infanticide dans la région de Québec (1660-1969)
}

\section{Marie-Aimée Cliche}

Volume 44, numéro 1, été 1990

URI : https://id.erudit.org/iderudit/304862ar

DOI : https://doi.org/10.7202/304862ar

Aller au sommaire du numéro

\section{Éditeur(s)}

Institut d'histoire de l'Amérique française

ISSN

0035-2357 (imprimé)

1492-1383 (numérique)

Découvrir la revue

Citer cet article

Cliche, M.-A. (1990). L'infanticide dans la région de Québec (1660-1969). Revue d'histoire de l'Amérique française, 44(1), 31-59. https://doi.org/10.7202/304862ar

\section{Résumé de l'article}

Depuis le début de la colonie jusqu'à nos jours, un certain nombre de jeunes filles ont eu recours à l'infanticide pour échapper au déshonneur d'une maternité hors mariage. Les 392 cas repérés dans les archives judiciaires du district de Québec révèlent que les circonstances entourant l'infanticide et les motivations des mères n'ont pas changé au cours de trois siècles, bien que leur nombre aille en diminuant. L'attitude des juges, par contre, évolue énormément, passant d'une sévérité impitoyable sous le Régime français à une attitude de clémence pleine de paternalisme à partir du XIX ${ }^{\mathrm{e}}$ siècle. On relève les mêmes traits dans d'autres régions, notamment en Ontario, ce qui dénote des comportements semblables dans les sociétés qui partagent les mêmes valeurs patriarcales. 


\title{
L'INFANTICIDE DANS LA RÉGION DE QUÉBEC $(1660-1969)^{1}$
}

\author{
MARIE-AIMÉE CLICHE \\ historienne
}

\begin{abstract}
RÉSUMÉ
Depuis le début de la colonie jusqu'à nos jours, un certain nombre de jeunes filles ont eu recours à l'infanticide pour échapper au déshonneur d'une maternité hors mariage. Les 392 cas repérés dans les archives judiciaires du district de Québec révèlent que les circonstances entourant l'infanticide et les motivations des mères n'ont pas changé au cours de trois siècles, bien que leur nombre aille en diminuant. L'attitude des juges, par contre, évolue énormément, passant d'une sévérité impitoyable sous le Régime français à une attitude de clémence pleine de paternalisme à partir du XIXe siècle. On relève les mêmes traits dans d'autres régions, notamment en Ontario, ce qui dénote des comportements semblables dans les sociétés qui partagent les mêmes valeurs patriarcales.
\end{abstract}

\begin{abstract}
From the beginning of the colony to the present, a number of girls resorted to infanticide to escape the shame of illegitimate motherhood. The 392 cases located in the judicial archives of the district of Quebec show that, while the relative number of cases diminished, the circumstances surrounding infanticide and the mothers' motivations did not vary over three hundred years. On the other hand, there was a dramatic change in the attitude of magistrates, from merciless severity during the French Regime to compassion and paternalism from the beginning of the 19th century. Similar traits have been identified in other regions, particularly in Ontario, pointing to similar attitudes in societies which share the same patriarchal values.
\end{abstract}

\section{INTRODUCTION}

Parmi les faits divers portés à la connaissance du public, l'infanticide est l'un de ceux qui suscitent le plus de malaise dans la population. Car chacun se sent partagé entre des sentiments contraires: tout en étant bouleversé à l'idée du meurtre d'un nouveau-né, on hésite à condamner sans rémission la personne qui a posé ce geste, surtout lorsque c'est la propre mère de l'enfant.

1 Cette étude a été réalisée grâce à une bourse du Conseil de recherches en sciences humaines du Canada. Je remercie les personnes qui ont accepté de lire ce texte et de me faire part de leurs commentaires: les historiens Jacques Gélis, de l'Université de Paris VIII, Jacques Mathieu, de 1'Université Laval, et Ann Robinson, professeure de droit à l'Université Laval, ainsi que les évaluateurs et le Comité de rédaction de la Revue d'histoire de l'Amérique française. 
Mais en a-t-il toujours été ainsi? Dans l'antiquité, l'abandon des nouveau-nés était une pratique communément admise et même au Moyen Âge, la suppression d'enfants, surtout ceux du sexe féminin, s'est maintenue dans certaines régions ${ }^{2}$. Ce n'est que graduellement, grâce à l'amélioration du niveau de vie et à la connaissance de méthodes contraceptives et abortives sûres, que ces coutumes se raréfièrent au point de devenir des faits exceptionnels.

Le christianisme a toujours considéré l'infanticide comme un crime, d'autant plus grave que les enfants morts sans baptême sont privés du bonheur céleste. C'est à son influence et à son action que l'on doit les premières mesures prises pour lutter contre cette coutume, soit la création d'hospices pour accueillir les enfants abandonnés et la promulgation de lois pour punir les infanticides ${ }^{3}$.

Plusieurs historiens ont traité cette question, tant en Europe qu'en Amérique, à différentes périodes. Leur objectif est de découvrir les motivations des personnes accusées, l'attitude des juges à leur endroit et l'évolution des lois. Ils constatent l'origine sociale modeste des accusées, insistent sur le sort pénible fait aux filles-mères dans les sociétés patriarcales, et soulignent la clémence manifestée par les tribunaux à partir du début du XIXe siècle ${ }^{4}$. Notons en particulier l'étude de Cons-

2 William L. Langer, «Infanticide: a Historical Survey», History of Childhood Quarterly, 1,3 (hiver 1974): 353-365; Jacques Gélis, «Le refus du nouveau-né», L'arbre et le fruit. La naissance dans l'Occident moderne XVIe-XIXe siècle (Paris, Fayard, 1984), 404-433; Jean-Louis Flandrin, «L'attitude à l'égard du petit enfant et les conduites sexuelles», Le sexe et l'Occident (Paris, Seuil, 1981), 151-216; Y.-B. Brissaud, «L'infanticide à la fin du Moyen Age, ses motivations psychologiques et sa répression», Revue historique de droit français et étranger, 50 (1972): 229-256; Emily Coleman, «L'infanticide dans le Haut Moyen Age», Annales, Économie, Société, Civilisation, 29,2 (avril-mai 1974): 315-335; Barbara A. Kellum, «Infanticides in England in the Later Middle Ages», History of Childhood Quarterly, 1 (1974): 367-388.

3 L. Godefroy, «Infanticide», Dictionnaire de théologie catholique, 12 (1923): col 17171726; René Bouton, L'infanticide. Étude morale et juridique (Paris, Société d'édition scientifique, 1897), 74.

${ }^{4}$ La plupart des études réalisées jusqu'à présent sur l'histoire de l'infanticide sont basées sur les archives judiciaires, notamment: Constance B. Backhouse, «Desperate Women and Compassionate Courts: Infanticide in Nineteenth-Century Canada», University of Toronto Law Journal, 34 (1984): 447-478; George K. Behlmer, «Deadly Motherhood: Infanticide and Medical Opinion in Mid-Victorian England», Journal of the History of Medecine, 34,4 (octobre 1979): 403-427; Brissaud, op. cit.; D. Seaborne Davies, "Child-Killing in English Law», Modern Law Review, 1 (décembre 1937): 203-223; Dr Fauvelle, «Contribution à l'étude anthropologique de l'infanticide», L'Homme (1885): 328-334; Paul A. Gilje, «Infant Abandonment in Early Nineteenth Century New York City: three Cases», Signs, 8,3 (printemps 1983): 580-590; P. E. H. Hair, «Homicide, Infanticide and Child Assault in Late Tudor Middlesex», Local Population Studies, 9 (1972): 43-48; René Leboutte, «L'infanticide dans l'est de la Belgique aux XVIIleXIXe siècles: une réalité», Annales de démographie historique (1983): 163-192; R. W. Malcolmson, «Infanticide in the Eighteenth Century», J. S. Cockburn, ed., Crime in England, 1550-1800 (Princeton (New Jersey), Princeton University Press, 1978), 187-209; Alain Vlamynck, «La délinquance au féminin: crimes et répression dans le Nord, 1880-1913», Revue du Nord, 63,250 (juillet-septembre 1981): 675-702; Nigel Walker, «Infanticide», Crime and Insanity in England (Edinburgh, Edinburgh University Press, 1968), 1: 125-137; W. Peter Ward, "Unwed Motherhood in Nineteenth-Century English Canada», Communications historiques, Société historique du Canada (1981): 34-56; Keith Wrightson, «Infanticide in Earlier Seventeenth-Century England», Local Population Studies, 15 (1975): 10-20. 
tance Backhouse qui porte sur l'Ontario au XIXe siècle où les lois criminelles étaient les mêmes qu'au Québec. Son titre est particulièrement évocateur: «Desperate Women and Compassionate Courts». Elle voit dans l'infanticide un geste de révolte posé par des femmes décidées à contrôler leur destin, et dans la clémence des juges une preuve que la société respectait leur décision ${ }^{5}$.

Il n'existe pas encore d'études sur l'infanticide au Québec. Dans la foulée des autres historiens, nous avons abordé ce sujet dans une double perspective: d'abord, essayer de comprendre les motivations du geste meurtrier à la lumière des circonstances qui l'entourent et des explications fournies par les responsables; ensuite, découvrir l'attitude des hommes de loi au moyen des verdicts et des sentences qu'ils rendent et des raisons qu'ils font valoir. Nous essaierons en particulier de saisir comment les valeurs patriarcales se manifestent dans le cours des procédures judiciaires $^{6}$. A travers ces dossiers, c'est toute la mentalité de la société québécoise à une époque donnée que nous rejoignons. Nous avons choisi de traiter ce sujet sur une très longue période dans le but de faire ressortir les traits durables du phénomène et les changements qu'il a subis.

Notre documentation est tirée des archives judiciaires du district de Québec, en particulier les rapports du coroner, ce fonctionnaire chargé d'enquêter sur tous les cas de mort suspecte, y compris celle des enfants nouveau-nés. Un verdict de mort criminelle entraîne un procès (quand le coupable est connu) dont les actes sont généralement conservés ${ }^{7}$.

5 Backhouse, op. cit. Ann Jones, Women Who Kill (New York, Holt, Rinehart and Winston, 1980), 51 .

6 Voir à ce sujet les études suivantes: Marie-Andrée Bertrand, La femme et le crime (Montréal, L'Aurore, 1979); Christiane Boyle et al., Un examen féministe du droit criminel (Ottawa, Condition féminine, 1985), 158-159; Arlette Farge et al., "Culture et pouvoir des femmes: essai d'historiographie», Annales ESC (mars-avril 1986): 283; Odile Dhavernas, Droits des femmes, pouvoir des hommes (Paris, Seuil, 1978).

7 Archives nationales du Québec (ANQ): nous avons dépouillé systématiquement les fonds suivants: Enquêtes du coroner (EC), 1765-1948; ces documents sont rattachés aux archives de la Cour supérieure (CS) pour la période 1765-1930, et à la Cour des Sessions de la paix (CSP) pour la période 1931-1948, avec certaines exceptions; Cour supérieure de Québec, section criminelle, 1765-1925 (je remercie mon collègue Jean-Marie Fecteau qui m'a indiqué les cas d'infanticide dans ces dossiers); Cour des Sessions de la paix (CSP), 1802-1948; Cour des Sessions de la paix, instruction préliminaire (IP), 1896-1921; Registres de la prison de Québec, 1813-1962; Département du Procureur général (DPG), 1867-1937; nous avons dépouillé ce fonds en nous limitant à un échantillon, soit l'année 7 de chaque décennie. Centre de préarchivage de Québec (Préarch.): Cour des Sessions de la paix, 1949-1969; Enquêtes du Coroner, 1949-1969 (je remercie ma collaboratrice Louise Poitras qui a dépouillé ces fonds avec moi). Archives de la Maison Généralice des Soeurs du Bon-Pasteur de Québec (AMGBP): nous avons dépouillé les dossiers des pensionnaires des institutions suivantes: Maison Sainte-Madeleine (MSM), 1910-1972; Maison Notre-Dame de la Garde (MNDG), 1945-1973; nous remercions les religieuses de cette communauté de nous avoir permis de consulter leurs archives. Pour la période 1760-1969, nous avons limité nos recherches au district judiciaire de Québec, mais la correspondance du Procureur général rapporte des cas qui se sont déroulés dans toute la province; pour la période du Régime français, nous avions dépouillé toutes les archives judiciaires des gouvernements de Québec, de Montréal et de Trois-Rivières; nos tableaux contiennent uniquement les données concernant la région de Québec. 
Ces sources ne permettent pas d'évaluer l'ampleur du phénomène de l'infanticide, comme l'ont souligné plusieurs chercheurs ${ }^{8}$. D'abord, parce qu'une partie des archives du coroner ont visiblement disparu, surtout pour les XVIIIe et XIXe siècles, ainsi qu'un certain nombre de procès. Ensuite, parce que la documentation ne rejoint que les cas qui sont portés à l'attention du coroner et éventuellement des juges. Si la méthode statistique ne peut produire que des résultats imparfaits, ces documents offrent en revanche d'autres possibilités, car ils sont d'une grande richesse du point de vue qualitatif. Les rapports d'autopsie, les déclarations des policiers et des témoins ainsi que les aveux des personnes inculpées aident à reconstituer les circonstances entourant la mort de l'enfant, tandis que les sentences rendues par les juges permettent de suivre l'évolution de la jurisprudence. Mais il y a plus et mieux. Le discours énoncé par les personnes accusées qui tentent d'expliquer leur geste et de se justifier, les propos tenus par les membres de leur entourage qui essaient d'écarter tout soupçon de complicité, enfin les raisons avancées par le juge pour soutenir son verdict, tout cela nous permet d'atteindre notre objectif, soit éclairer les mentalités.

\section{1 - TUER OU LAISSER MOURIR}

Un nouveau-né est un être fragile. Les soins accordés ou refusés dans les moments qui suivent la naissance entraînent sa survie ou son trépas sans qu'il soit nécessaire de recourir à des moyens violents. Et quand la vie s'est éteinte, il est relativement facile de dissimuler un corps aussi petit. Cependant, une femme qui vient d'accoucher n'a généralement pas la force d'aller le porter bien loin (à moins que quelqu'un d'autre s'en charge) et il n'est pas toujours possible de garder secrets une grossesse et un accouchement. Un certain nombre de cadavres ont donc été retrouvés, ce qui a donné lieu à des enquêtes plus ou moins élaborées qui révèlent parfois les circonstances de la mort.

Le rapport entre le nombre exact d'infanticides et les cas qui font l'objet d'une enquête nous échappe, aussi faut-il interpréter les chiffres du tableau 1 avec prudence. Il semble bien cependant que le nombre relatif, en regard de la croissance de la population de la région de Québec, diminue progressivement. Les variations décennales ne sont pas nécessairement significatives, mais certaines coïncidences sont quand même frappantes. Ainsi, l'augmentation durant la décennie 1860 qui se produisit à la fois au Québec et en Angleterre dépend peut-être d'une modification des conditions de travail du coroner, à qui on demande de multiplier les enquêtes ${ }^{9}$. D'autre part, nous sommes tentée de relier

\footnotetext{
8 Dans le présent texte, nous emploierons le mot «infanticide, dans le sens suivant: le fait de causer la mort d'un enfant nouveau-né, par action ou par omission. À partir de 1948, la loi donnera un sens plus restrictif à ce terme. Malcolmson, op. cit., 191; Leboutte, op. cit., 179; Backhouse, op . cit., 456; R. Sauer, 'Infanticide and Abortion in Nineteenth-Century Britain», Population Studies, 32,1 (mars 1978): 81.

9 Backhouse, op . cit., 456; Behlmer, op. cit., 406.
} 
l'augmentation d'infanticides dans la décennie 1930 à la crise économique. Quant à la baisse prononcée pendant la période suivante, surtout après 1960, il nous paraît plausible de l'attribuer à la diffusion des méthodes contraceptives et peut-être des pratiques abortives.

TABLEAU 1

Procédures judiciaires à la suite de la découverte de cadavres de nouveau-nés dans le district de Québec 1660-1969

\begin{tabular}{|c|c|c|c|c|}
\hline Périodes & $\begin{array}{c}\text { Nombre } \\
\text { d'enquêtes du } \\
\text { coroner }\end{array}$ & $\begin{array}{c}\text { Verdict de } \\
\text { mort criminelle } \\
\text { (coroner) }\end{array}$ & $\begin{array}{c}\text { Nombre de cas } \\
\text { où la mère } \\
\text { est connue }\end{array}$ & Procès \\
\hline $1660-1759$ & $(4)$ & 4 & 4 & 4 \\
$1760-1809$ & 2 & 2 & 2 & 2 \\
$1810-1819$ & 6 & 3 & 4 & 2 \\
$1820-1829$ & 12 & 2 & 6 & 3 \\
$1830-1839$ & 8 & 2 & 3 & 2 \\
$1840-1849$ & 23 & 5 & 8 & 10 \\
$1850-1859$ & 34 & 12 & 12 & 2 \\
$1860-1869$ & 50 & 7 & 14 & 5 \\
$1870-1879$ & 32 & 8 & 8 & 2 \\
$1880-1889$ & 20 & 9 & 4 & 4 \\
$1890-1899$ & 11 & 2 & 4 & 2 \\
$1900-1909$ & 20 & 11 & 7 & 9 \\
$1910-1919$ & 19 & 6 & 17 & 9 \\
$1920-1929$ & 36 & 18 & 33 & 7 \\
$1930-1939$ & 52 & 10 & 17 & 5 \\
$1940-1949$ & 33 & 10 & 11 & 2 \\
$1950-1959$ & 21 & 6 & 5 & 75 \\
$1960-1969$ & 9 & 1 & 167 & 4 \\
& 392 & 118 & 5 & 2 \\
\hline
\end{tabular}

Source: ANQ et Préarch., voir note 6.

Les trois quarts des petits cadavres furent retrouvés dans les villes de Québec et de Lévis ( 275 cas sur les 367 connus) et les autres dans les paroisses rurales. Mais n'allons pas en conclure tout de suite que l'infanticide était un phénomène surtout urbain, car nous savons qu'une partie des enfants remis aux hospices de Québec venaient de la campagne et nous ignorons tout de l'origine de ceux abandonnés dans des endroits publics.

Plus de la moitié des nouveau-nés sont retrouvés dans des endroits publics ${ }^{10}$. C'est probablement dans l'espoir qu'ils puissent être recueil-

10 Les 392 cas se répartissent comme suit: retrouvés dans une église, un cimetière ou les environs, 48; près d'un hospice, 24 ; sur la grève, près d'un quai ou dans l'eau, 35 ; dans d'autres 
lis vivants que des personnes les abandonnent près d'un hospice, et pour leur assurer une sépulture religieuse que d'autres les laissent dans une église ou un cimetière. Il y a peut-être parmi ces derniers des enfants légitimes de parents pauvres qui veulent éviter les frais d'inhumation ${ }^{11}$. Mais dans la plupart des cas, le lieu traduit le désarroi de la mère qui accouche en cachette et abandonne l'enfant sur place ou tente de le dissimuler n'importe où.

La cause de la mort est parfois évidente, surtout si on trouve le petit cadavre flottant sur les eaux ou gelé dans la neige. Les rapports d'autopsie fournissent des informations plus détaillées sur les moyens violents employés pour causer la mort (fracture du crâne, strangulation, égorgement, suffocation) ou sur le manque de soins qui a abouti au même résultat (tableau 2). Cependant, le coroner se montre très prudent dans l'énoncé de ses conclusions, pour des raisons que nous verrons plus loin. Le verdict de mort criminelle n'est prononcé que dans une minorité de cas (voir tableau 1).

\section{TABLEAU 2}

Causes de la mort des enfants trouvés

1660-1969

Fracture du crâne

Strangulation

Égorgement

Suffocation

Noyade

Exposition au froid

Manque de soins

Hémorragie

Asphyxie

Mort naturelle ou mort-né

Cause inconnue

TOTAL

Source: ANQ et Préarch., EC.

La différence est souvent bien mince entre une mort naturelle, un accident et un assassinat. Il ne fait pas de doute que certains décès peuvent être dus à des causes naturelles. Après tout, certains enfants naissaient morts ou non viables, même quand la mère était entourée de soins.

D'autres décès peuvent être considérés comme des accidents causés par l'imprudence ou la négligence. Par exemple, lorsqu'un nouveau-né est abandonné dans un lieu public, sa vie ne tient qu'à un fil. Si l'endroit

endroits publics, 49; dépotoirs et poubelles, 14; dans les champs ou les bois, 23; dans une maison ou des dépendances, 59; lieu inconnu, 140. ANQ et Préarch., EC.

11 On signale cette façon d'agir en Belgique et en Angleterre. Leboutte, op. cit., 167; Behlmer, op. cit., 425. ANQ, CSP, EC, ler mars 1933. Un nouveau-né est trouvé au coin d'une rue, dans une boîte, avec ce billet: «M. le curé, seriez-vous assez bon d'enterrer le bébé. (...) C'est mon mari qui est allé le porter dans votre église.» ANQ, CS, EC, 27 juin 1879. Un vieil homme illettré est chargé de porter au cimetière son petit-fils, mort-né. «Ne connaissant pas mieux», il dépose le petit cadavre dans la première fosse ouverte qu'il voit et repart sans en parler au fossoyeur. Le bedeau et le curé demandent évidemment au coroner d'effectuer une enquête. 
est achalandé, il a des chances d'être recueilli rapidement, comme cet enfant déposé près de l'entrée d'un hôtel en $1864^{12}$. Par contre, si on le laisse devant la porte fermée d'un hôpital, sans signaler sa présence, par une nuit d'hiver, il risque fort de mourir de froid comme cela arrive en 1766, à l'Hôtel-Dieu, et en 1882, à l'hôpital du Sacré-Coeur ${ }^{13}$.

Parfois, ces abandons s'accompagnent de précautions qui témoignent du désir de conserver la vie de l'enfant. Durant l'été 1914, les religieuses trouvent à la porte de la Crèche un bébé nouveau-né, placé dans une boîte à chaussures, avec deux biscuits dans la main ${ }^{14}$. En 1940 , on découvre dans une chambre louée un bébé bien vêtu avec une «suce» attachée à sa main que ses parents avaient laissé là deux jours auparavant avec un billet demandant de s'en occuper. Presque mort d'inanition quand la propriétaire de la maison le découvre, il meurt peu après ${ }^{15}$. Dans plusieurs cas, on se demande si le décès doit être attribué à l'ignorance ou à la malveillance. Par exemple, ces enfants enveloppés trop étroitement et qui meurent étouffés avant d'arriver à l'hôpital ${ }^{16}$, ou ceux qui succombent à une hémorragie parce que le cordon ombilical n'a pas été attaché ${ }^{17}$. Ceux, enfin, qui meurent suffoqués dans les toilettes parce que la mère aurait confondu les douleurs de l'accouchement avec la colique (11 cas).

Dans presque la moitié des cas, les autorités ont pu identifier la mère. Il s'agit de 149 célibataires, 11 femmes mariées et 7 veuves. Nous ne saurions dire combien d'enfants d'origine inconnue sont issus de parents légitimement mariés, mais lors des enquêtes, les soupçons portent d'abord sur les filles-mères ${ }^{18}$. Elles ont certainement plus de raisons et moins de facilités que d'autres pour dissimuler une naissance. Les corps de ces nouveau-nés sont retrouvés plus souvent entre les mois de février et mai qu'en d'autres saisons ${ }^{19}$. Or cela correspond sensiblement avec la période où les accouchements des filles-mères sont le plus

\footnotetext{
12 ANQ, CSP, b. 154, 4 mars 1864. 1882

ANQ, CS, EC, 3 février 1766; CS, crim., février-juin 1766; ANQ, CS, EC, 17 février

i4 Archives de la Crèche Saint-Vincent-de-Paul, Annales, 1: 268, 9 juillet 1914.

15 ANQ, CSP, EC, 5 janvier 1940.

16 ANQ, CS, EC, 22 juillet 1907.

17 À vrai dire, dès le XIXe siècle, les médecins émettaient l'opinion que le fait de ne pas ligaturer le cordon ombilical entraînait rarement une hémorragie mortelle. Mais de nombreuses enquêtes du coroner conclurent que l'enfant était mort parce que le cordon n'avait pas été ligaturé. Jos. Briand et Ernest Chaudé, Manuel complet de médecine légale (Paris, Baillière, 1879), 386387. J. J. Desmarez, Manuel de médecine légale à l'usage des juristes (Paris, Presses universitaires de France, 1967), 447.

18 ANQ, Montréal, Documents judiciaires, b. 71, 12-13 juin 1723. Le procureur du roi fait une enquête sur le cas d'un enfant nouveau-né trouvé au bord de l'eau, la gorge tranchée. La première personne dénoncée et soupçonnée est une jeune fille. En 1927, à Bagotville, on trouve le cadavre d'un nouveau-né qui flotte sur la rivière Saguenay. Les policiers s'informent aussitôt au sujet de certaines filles qui ont eu des bébés. DPG, dossier 4249.

19 La répartition mensuelle est la suivante: janvier (25), février (44), mars (41), avril (46), mai (47), juin (26), juillet (27), août (27), septembre (23), octobre (18), novembre (18), décembre (17), inconnu (33).
} 
nombreux à l'Hôpital de la Miséricorde de Québec, entre 1913 et $1946^{20}$. Il y a là un indice de l'illégitimité des enfants nés de mère inconnue.

Les données statistiques concernant l'infanticide et les circonstances qui entourent ces décès dans la région de Québec, entre le XVIIIe siècle et 1969 , ressemblent énormément à celles qui ont été recueillies dans les autres pays, ce qui reflète probablement des coutumes et des traits de mentalité semblables. Cet aspect de la question peut être éclairé à la lumière des propos tenus lors des enquêtes et des procès.

\section{2 - UN DISCOURS JUSTIFICATIF}

Les personnes soupçonnées d'infanticide qui comparaissent devant le coroner ou le juge sont des jeunes filles dans 9 cas sur 10. Lorsque leur âge et leur métier sont indiqués, on constate que les trois quarts d'entre elles ont moins de 25 ans et gagnent leur vie comme servantes ${ }^{21}$. Si elles ont reçu l'aide de quelqu'un dans leur malheur, c'est celle de leur amant ou d'un proche parent, mais le fait est plutôt rare. Dans 12 cas seulement voit-on apparaitre un complice à la barre des accusés ${ }^{22}$.

Quelles que soient les personnes soupçonnées, le type d'argument employé pour se justifier ne varie guère. Presque toutes affirment que l'enfant est mort-né ou décédé peu après sa naissance sans qu'elles aient rien fait pour hâter l'issue fatale. Par exemple, Alice A., une servante qui accoucha seule en 1924, dit qu'elle resta assise par terre pendant une heure «ayant l'enfant entre mes jambes et l'arrière-faix dans ma matrice», trop affaiblie pour s'occuper du nouveau-né qui ne bougeait pas. Béatrice B., une autre servante, qui laissa son enfant sur le sol toute la nuit, avant de le placer dans une valise, déclare: «Je n'ai rien fait à l'enfant [...] Je ne l'ai seulement pas regardé [...] Je n'ai pas pensé à l'empêcher de respirer.» Toutes deux admettent encore avoir caché le cadavre pour dissimuler la naissance ${ }^{23}$. Il leur reste à expliquer pourquoi elles ont agi de la sorte.

Les explications fournies dans de telles circonstances peuvent être véridiques, exagérées ou absolument fausses. Elles peuvent aussi être dictées par un avocat. Mais elles doivent toujours conserver une cer-

\footnotetext{
20 ASBP, Miséricorde (9), boîte 18, chemise 2. Rythme mensuel des accouchements, 18741972.

Les 86 filles dont l'âge est connu se répartissent comme suit: 16 à 19 ans (23), 20 à 24 ans (43), 25 à 29 ans (16), 30 à 39 ans (3), 40 à 49 ans (1). Parmi les 59 filles dont le métier est connu, on compte 46 servantes, 2 couturières, 3 ouvrières, 1 serveuse, 1 commis, 1 dactylo, 1 institutrice, 2 ménagères, 2 étudiantes.

${ }_{22}$ Parmi les 75 procès, on compte 63 cas où la mère seule est accusée, 6 cas où elle est accusée avec des complices, 6 cas où elle n'est pas accusée. Les complices sont l'amant ( 5 cas), les proches parents ( 5 cas) ou d'autres personnes ( 2 cas).

23 ANQ, CS, EC, b. 2347, no 109, 11 mars 1924; CSP, EC, b. 15, no 3135, 8 mars 1936. Pour des raisons de discrétion évidentes, nous ne citerons pas les noms réels des personnes soupçonnées d'infanticide, sauf pour le Régime français. Nous avons attribué à toutes les autres des prénoms fictifs, choisis par ordre alphabétique, à consonance anglaise ou française selon le cas.
} 
taine vraisemblance qui les rende acceptables par les hommes de loi. Comme telles, elles sont révélatrices des valeurs d'une société.

\section{1 - Cacher sa honte}

Parmi toutes les explications, une ressort avec une évidence particulière, soit le désir de cacher une naissance illégitime qui déshonore une jeune fille et toute sa famille.

Il existe différents moyens pour atteindre ce but sans attenter à la vie de l'enfant. À partir de 1852, les filles peuvent accoucher discrètement à la Miséricorde de Québec, institution fondée justement dans le but de prévenir les infanticides et «éloigner le déshonneur des familles respectables». Autre solution, la fille-mère peut accoucher dans sa famille puis envoyer l'enfant dans un hospice à Québec ${ }^{24}$. Mais le voyage s'avère fatal pour le nouveau-né dans onze cas au moins. L'un d'entre eux, un enfant prématuré, meurt dans le train entre Newport et Sherbrooke en 1892; un autre, également prématuré meurt entre Matane et Québec en $1920^{25}$. Pourquoi imposer de tels voyages à des enfants si fragiles? «Pour sauver l'honneur de la mère», répond la grand-mère du premier. La même explication est fournie par Céline C. qui apporte à Québec, en mars 1902, l'enfant de sa soeur né près de Lévis. Soucieuse de préserver la réputation de sa famille, elle dit aux religieuses de l'Hôpital du Sacré-Coeur que la mère est une fille d'une paroisse voisine. C'est seulement lors de l'enquête du coroner, à la suite du décès de l'enfant attribué à l'exposition au froid, qu'elle avoue le lien de parentée ${ }^{26}$.

Ce désir de garder secrète l'identité de la fille-mère et de sa famille occasionne bien d'autres décès. En février 1882, la mère de Diane D. apporte à l'Hôpital du Sacré-Coeur l'enfant de sa fille, le dépose sur le seuil, repart sans plus attendre, et l'enfant meurt de froid durant la nuit $^{27}$. En tout, 24 enfants furent ainsi trouvés morts près d'un hospice. Pour éviter de tels accidents, les religieuses installent différents systèmes permettant de recueillir les enfants de façon sécuritaire tout en respectant l'anonymat du porteur. L'Hôtel-Dieu possède un «tour», sorte de cylindre pivotant. Tout en restant à l'extérieur, il est possible de déposer un enfant dans ce cylindre et de le faire tourner pour qu'il

24 Une maternité fut fondée en 1852 par une laïque, Marie Métivier, et portait alors le nom d'Hospice Saint-Joseph. Elle passa sous le contrôle des Soeurs du Bon-Pasteur en 1874 et prit alors le nom d'Hôpital de la Miséricorde. Henri-Raymond Casgrain, L'asile du Bon-Pasteur de Québec (Québec, Demers, 1896), 268. Trois hôpitaux de Québec accueillirent tour à tour les enfants abandonnés: l'Hôtel-Dieu, de 1800 à 1850; l'Hôpital du Sacré-Coeur, de 1873 à 1901; la Crèche Saint-Vincent-de-Paul, à partir de 1901, accueillit les enfants qui naissaient à la Miséricorde et ceux qu'on apportait de l'extérieur. Voir l'étude du docteur de La Broquerie Fortier, «Les enfants trouvés sous les Régimes français et anglais au Canada français, 1608-1850", Laval médical, 33 (septembre 1962): 530-537; 34 (avril 1963): 442-453; (décembre 1963): 1242-1254; (mars 1964): 335-347; (avril 1964): 469-480; 36 (avril 1965): 351-359; (mai 1965): 466-476.

25 ANQ, CS, EC, 14 septembre 1892 et 17 mars 1920.

26 ANQ, CS, Crim, 11 avril 1902.

27 ANQ, CS, EC, 17 février 1882 
se retrouve à l'intérieur de la maison. À l'Hôpital du Sacré-Coeur, on peut remettre l'enfant par un guichet à une employée qui veille au parloir. A la Crèche Saint-Vincent-de-Paul, les religieuses installent un berceau dans un endroit chauffé, entre deux portes, pour recevoir les nouveau-nés.

Mais plutôt que d'avoir recours à une personne de leur entourage pour aller porter l'enfant à l'hospice, plusieurs filles-mères préfèrent garder leur secret à tout prix, et accoucher seules. Huit d'entre elles mettent leur enfant au monde dans un endroit désert: hangar, bois, etc.; 50 autres dans une maison, mais en se cachant de tous. Parmi ces 58 cas, 46 naissances sont suivies d'un infanticide. Par contre, lorsque la parturiente est assistée par quelqu'un (47 cas), la mort immédiate de l'enfant est beaucoup plus rare: 8 cas seulement. Au Québec comme partout ailleurs, l'infanticide est essentiellement un crime de la solitude $^{28}$. Tout se joue dans les quelques secondes qui suivent la naissance. Il suffit parfois de l'intervention d'une tierce personne pour empêcher de justesse la fille-mère de tuer l'enfant: «Ma mère voulait me tuer [...] quand je suis venue au monde, raconte une enfant illégitime, elle voulait aller me jeter à l'eau et c'est grand-maman qui l'a retenue. ${ }^{29}$ Élise E. accouche elle aussi dans la maison de ses parents, alors que sa mère est dans la pièce voisine, mais elle s'empresse de cacher dans une valise, «enterré dans du linge», l'enfant qui respire encore. «Je ne m'en suis pas occupée après. J'avais l'espérance qui meurt, afin que personne ne puisse s'en apercevoir.» ${ }^{30}$

À la crainte du déshonneur, s'ajoute celle des reproches et des sévices. Élise E. déclare: «Je n'avais rien osé dire à mes parents parce que $j$ 'avais peur que mon père me batte. ${ }^{31}$ Félicity $F$. dissimule également sa grossesse de crainte que sa mère ne la tue $\mathrm{e}^{32}$. Quant à Gail G., elle affirme avoir reçu des menaces de mort de son frère James qui était le père de l'enfant ${ }^{33}$. Certaines de ces déclarations peuvent être exagérées dans le but d'émouvoir le juge, mais elles ne sont sûrement pas dépourvues de fondement. Des parents se montraient très durs envers leur fille comme le montrent les lettres conservées dans les archives de l'Hôpital de la Miséricorde. Une travailleuse sociale rapporte également le cas d'une mère qui poursuivit sa fille de 17 ans avec une chaise

28 Malcolmson, op. cit., 194, 207; Vlamynck, op. cit., 682; Jones, op. cit., 45; Leboutte, op. cit., 182. M. Remacle, Rapport concernant les infanticides et les mort-nés dans leur relation avec la question des enfants trouvés (Paris, Imprimerie Royale, 1845), 18. «Sur 20 infanticides qui se commettent, il n'y en a pas un où la fille-mère, auteur du crime, n'ait éloigné d'elle, au moment de l'accouchement, les secours qui lui auraient été nécessaires.»

${ }_{29}$ AMGBP, MSM, 11-60. L'adolescente qui fait ce récit avait recueilli ces informations de la bouche de sa grand-mère.

30 ANQ, CSP, EC, 28 juillet 1932

31 ANQ, CSP, EC, b. 2, no 657, 28 juillet 1932.

32 ANQ, DPG, no 1395, mars-avril 1907.

33 ANQ, CS, EC, b. 2314, no 33, 3 avril 1858. 
et lui infligea une telle frayeur que cette dernière accoucha prématurément le même soir ${ }^{34}$.

Parfois, ce sont les parents eux-mêmes qui se chargent de disposer de l'enfant non désiré. En 1927, Madame H. va porter l'enfant de sa fille dans une petite chapelle, déclarant par la suite qu'il était mort-né. L'année suivante, un cultivateur est accusé d'avoir fait disparaître le cadavre de l'enfant de sa fille ${ }^{35}$. Dans d'autres cas, c'est apparemment l'amant de la jeune fille qui lui rend ce service. En 1851 et en 1853, Isidore I. et John J. sont accusés d'avoir déposé dans une étable et un fenil l'enfant de leur maîtresse ${ }^{36}$. Un siècle plus tard, un jeune homme va jeter le cadavre de l'enfant de son amie dans la rivière Saint-Charles, de crainte que son père le sache ${ }^{37}$.

À toutes les époques, on constate que la peur du déshonneur et des reproches des parents sont les principales raisons évoquées pour expliquer l'infanticide ou du moins la dissimulation de l'enfant.

\section{2 - La hantise de la misère}

Parmi les mères infanticides dont le métier est connu, les trois quarts sont des servantes. Or, pour trouver une place, il est très important qu'elles aient une bonne réputation. C'est l'idée exprimée par Céline C. qui apporte un enfant à l'Hôpital du Sacré-Coeur en 1902: «C'était pour tâcher d'abriller ma soeur, pour pas qu'elle ait trop de misère à gagner sa vie [...] parce qu'elle était obligée de s'engager en service.» ${ }^{38}$

L'honneur n'est pas le seul problème. Il est très difficile pour une fille-mère de faire son travail de servante tout en prodiguant à un nouveau-né les soins qu'il requiert. C'est le dilemme auquel est confrontée Kate K. en 1838. Après avoir accouché chez ses patrons, elle propose à sa maîtresse, Lucy L., de continuer son service tout en prenant soin de son enfant, mais cette dernière s'y oppose parce que "when she would be in the height of her work the child would be in her way and advised her to get rid of it. ${ }^{39}$ Après quoi, Madame L. fait venir une personne pour aller abandonner l'enfant dans un endroit public.

Ces deux arguments, la réputation de la mère et la charge de travail représentée par l'enfant, sont évoqués par Diane D. en 1882 pour justifier l'abandon de son enfant. Cette jeune fille vivait avec sa mère, une veuve qui travaillait à la journée.

34 Alice Fontaine, L'illégitimité et ses problèmes. Étude de vingt cas de filles-mères secourues par le bureau du Service social industriel des usines de Saint-Malo, thèse en service social, Université Laval, 1949, 5.

35 ANQ, CSP, b. 68, 13 octobre 1927; b. 245, no 448, 30 mai 1928.

36 ANQ, Cour Sup., crim, 6 mai 1853; Cour Sup., EC, 12 février 1851.

37 Préarch, CSP, EC, b. 1077, no 13938, 9 octobre 1953.

38 ANQ, CS, crim., b. 2299, no 3, 11 avril 1902.

39 ANQ, CSP, 2 mai 1838. 
Ma mère disait qu'il était impossible pour elle d'avoir soin de moi qui était malade au lit et en même temps d'avoir soin de mon petit enfant parce que elle était obligée de s'absenter tous les jours pour gagner sa vie. [...] Elle m'a dit qu'elle n'avait pas les moyens d'avoir soin et d'élever l'enfant et que ce serait mieux pour ma propre protection et celle de mon caractère de mettre l'enfant à l'hôpital [du Sacré-Coeur]. ${ }^{40}$

Certaines maîtresses de maison n'hésitent pas à congédier leurs servantes lorsqu'elles découvrent leur grossesse. C'est ce que fait Madame Cugnet à l'égard de Geneviève Gaudreau, en 1725. Cette dernière se réengage chez Madame Veillon, mais sans lui dire qu'elle attend un enfant, et elle le tue dès sa naissance. En 1821, Marguerite Grenier congédie également sa servante enceinte, malgré ses supplications ${ }^{\mathbb{1}}$.

La description de la servante qui accouche, en se cachant de tous, et fait ensuite disparaître son enfant, a été popularisée par la littérature et ensuite le cinéma. Que l'on songe à Adèle, dans Pot-Bouille de Émile Zola ou à Rosalie Prudent de Guy de Maupassant. Ce stéréotype colle à la réalité: les archives judiciaires de Québec contiennent une trentaine de cas qui correspondent exactement à ce scénario. Ce sont ceux qui touchaient le plus la sensibilité populaire. D'ailleurs, on les observe aussi en Ontario, en Angleterre et en Belgique, au XVIIIe et au XIXe siècles ${ }^{42}$.

\section{3 - La peur qui fait perdre la tête}

On comprend aisément que la situation pénible des filles-mères, la crainte du déshonneur, des reproches ou du congédiement leur inspirent parfois le désir de voir disparaître l'enfant qui leur cause tant de soucis. Quelques-unes expriment spontanément ce désir à une travailleuse sociale vers 1945. Apprenant sa grossesse, une jeune fille lui déclare: «Est-ce que cela est déjà arrivé qu'un enfant meure et ne naisse pas? Je vais commencer une neuvaine pour que cela se passe ainsi.» $\mathrm{La}$ même travailleuse sociale cite le cas de trois autres filles-mères qui demandaient à la Vierge de venir chercher leur enfant avant leur départ de l'hôpital afin qu'elles n'en aient pas d'inquiétude ${ }^{43}$. Une dernière exprima le désir que Dieu vint chercher son enfant «après le baptême, à sa naissance». Ainsi, elle serait certaine qu'il irait au $\operatorname{ciel}^{44}$. Fait à

40 ANQ, CS, EC, b. 2318, no 2, 17 février 1882.

41 ANQ, Collection de pièces judiciaires et notariales, nos 730 et 739, janvier 1726; CS, EC, b. 2303, no 6, 21 mars 1821 .

Émile Zola, Pot-Bouille (Fasquelle, 1957 [1882]), 412-418. Guy de Maupassant, «Rosalie Prudent», Contes et Nouvelles, 1884-1890 (Paris, Laffont, 1988), 806-809. Voir aussi, Malcolmson, op. cit., 192, 202, 207; Behlmer, op. cit., 420; Backhouse, op . cit., 457; Leboutte, op. cit., 181; Vlamynck, op. cit., 691

43 Fontaine, op. cit., 7 et 25

44 AMGBP, MNDG, 528-59. 
souligner, aucune de ces cinq filles qui souhaitent voir mourir leur enfant ne passe des paroles aux actes. Au contraire, toutes tentent d'assurer sa survie. Il semble que le fait d'extérioriser en le verbalisant leur désir de mort, suffit à l'exorciser. Les filles-mères qui tuent leur enfant sont celles, au contraire, qui cachent leur grossesse, qui n'en parlent à personne.

Il est assez facile de comprendre le comportement d'une fille-mère qui, après avoir dissimulé sa grossesse, supprime l'enfant et fait disparaître le corps pour qu'il ne subsiste nulle trace de l'événement. Mais il est moins aisé de comprendre l'attitude de Marguerite $\mathbf{M}$. qui jette le nouveau-né par la fenêtre dans une cour commune, ou celle de Nadine N. qui le dépose tout simplement dans la neige près de la porte d'entrée ${ }^{45}$. Pourquoi laisser le corps ainsi exposé à la vue de tous?

La psychanalyse moderne a fouillé ce comportement incompréhensible à première vue:

Chez la femme enceinte célibataire, refusée par l'homme, par la famille, par la société, le foetus reste dans la phase de symbiose physiologique; il est vécu par elle comme une chose que l'on doit ou qu'on peut cacher. Dans certains cas, la forme du corps de la femme enceinte célibataire reflète son état psychique au point de ne pas rendre évidente la grossesse. Ce sont les cas où, à l'échéance du neuvième mois, l'accouchement se produit à l'improviste, comme si le corps de la femme se libérait d'un corps étranger; ce sont les cas parmi lesquels se vérifie l'infanticide. [...] Cette femme ne tue pas une créature, car elle n'a pas eu la possibilité de la vivre comme telle; la symbiose est restée physiologique. ${ }^{46}$

Cette explication s'applique bien à un certain nombre de fillesmères infanticides. Pour une vingtaine d'entre elles, la grossesse, sans doute dissimulée par leur embonpoint habituel, ne paraît absolument pas. Il est vrai que leurs proches peuvent prétendre n'avoir rien remarqué pour écarter tout soupçon de complicité, mais la bonne foi de certains parents ne fait pas de doute. La mère d'Odile $\mathrm{O}$., par exemple, exprime la surprise qui la saisit en trouvant sa fille «encantée au pied du lit, tout habillée, [qui] venait d'accoucher, une petite fille entre les jambes» ${ }^{47}$. Certains accouchements étaient étonnamment rapides aussi, surtout pour des primipares. Pearl P. et Rita R. accouchent en pleine rue ${ }^{48}$. Simone $S$. et Thérèse $T$. ont tout juste le temps de se réfugier dans un hangar ${ }^{49}$.

Certaines filles nient également leur grossesse avec obstination même quand le médecin lui-même leur en apporte la confirmation. En

45 ANQ, CSP, EC, 14 février 1941; CSP, b. 276, no 3406, 21 février 1930.

46 Elda Scarzella, «La mère célibataire et son enfant. Italie», Informations sociales, 22,12 (janv.-fév. 1968): 53

47 ANQ, CS, EC, 24 octobre 1895.

48 ANQ, CS, EC, 17 mars 1862 et 4 mai 1914.

49 ANQ, CSP, EC, 2 décembre 1933 et 5 avril 1937. 
1875 par exemple, à Saint-Nicolas, le docteur Morin propose à Ursule U. de l'envoyer dans une maternité à Québec, où elle serait bien soignée et pourrait accoucher sans scandale. Mais la jeune fille persiste à nier. Le moment de l'accouchement venu, elle se retire dans un bois puis dissimule l'enfant sous un tas de feuilles ${ }^{50}$. Ne peut-on pas voir dans ces dénégations une tentative pour fuir une réalité insupportable? En 1962, une autre fille déclare qu'elle n'avait pas compris vraiment qu'elle était enceinte. "C'est seulement à l'accouchement que je le réalisais, même si je ne le croyais pas. Ça m'a donné un choc.» Une autre dit: «Tout s'est passé comme dans un rêve.» ${ }^{51}$ Cet état de choc, de demiconscience, est évoqué maintes fois par des filles-mères pour expliquer leur geste meurtrier. En 1726, Geneviève Gaudreau dit que «elle était toute émue [...] et si transportée qu'elle ne savait pas ce qu'elle faisait alors.» Pearl P. dit elle aussi qu'elle était «trop faible et trop troublée, qu'elle ne savait pas ce qu'elle faisait». Élise E. et Vivianne V. évoquent la même excuse: «J'étais faible et énervée» et une dernière déclare en 1956: «J'étais comme une folle. J'ai perdu la carte. $»^{52}$

Bref, les raisons exposées par les filles-mères infanticides pour expliquer leur geste ne varient pas entre le XVIIIe et le XXe siècle. Sans doute, au cours de cette période, le nombre de grossesses illégitimes a augmenté en même temps que la population, de plus les moyens de les dissimuler se sont améliorés avec la fondation de crèches et d'hospices. Mais pour la fraction de filles-mères qui, pour une raison ou une autre, n'ont pas recouru à ces services, la peur et la honte sont aussi fortes à la fin qu'au début de la période. Le déroulement des procès nous révèle comment la société, représentée par les membres de l'appareil judiciaire, réagit à cette situation.

\section{3 - LE JUGEMENT DES HOMMES}

Pendant la période de trois siècles que nous étudions, les coutumes judiciaires ont connu des transformations profondes. Il y a loin des condamnations à mort pour vol du XVIIe siècle, aux procès du XXe, accompagnés d'expertises médicales et psychiatriques. L'évolution de la jurisprudence, de même que les commentaires formulés au fil du temps au cours des enquêtes et des procès, permettent de saisir les changements dans la mentalité des hommes de loi et partant dans celle de la société à laquelle ils appartiennent.

\section{1 - Les lois de l'Ancien régime: faire un exemple}

Les lois de l'Ancien régime présentaient deux caractères qui sont étrangers au système pénal actuel. Les peines prévues pour les délits

50 ANQ, CS, EC, b. 2316, no 36, 2 juillet 1875.

51 AMGBP, MSM, 83-62 et 34-68.

52 ANQ, CPJN, no 730, 1726; CS, EC, 17 mars 1862; CSP, EC, 28 juillet 1932 et 13 mai 1935; Préarch., CSP, EC, 25 novembre 1956. 
criminels étaient très sévères, souvent cruelles et exécutées en public dans le but d'exercer un effet dissuasif sur la population. De plus, les accusés étaient soumis à la torture pour leur arracher des aveux. Nous retrouvons la première de ces particularités dans le fameux Édit d'Henri II, destiné à réprimer les infanticides: toute femme qui cache sa grossesse et son accouchement et dont l'enfant meurt, est tenue responsable de ce décès et punie de mort, «afin que ce soit exemple à tous» ${ }^{33}$.

Dans l'ensemble du Canada, sous le Régime français, sept femmes sont accusées d'infanticide dont trois sont mises à mort ${ }^{54}$. Les juges basent leur verdict sur trois considérations: constatation du crime, découverte du coupable, connaissance de la loi par l'accusée ${ }^{55}$. Les filles qui répondent à ces conditions sont systématiquement condamnées à la potence, mais il arrive que la peine soit ensuite commuée. Dans le gouvernement de Québec, quatre femmes subissent un procès pour infanticide avant 1760, mais une seule, Marie-Anne Sigouin, est exécutée en 1732. Marie-Barbe Dupont veuve Pinel qui s'obstine à nier les faits est torturée mais a finalement la vie sauve ${ }^{56}$. Le but des hommes de loi est de faire un exemple, comme l'atteste une lettre de l'intendant Hocquart au Ministre: «J'espère que l'exemple que le Conseil Souverain a fait dans les personnes de Marie-Anne Gendron et Anne Sigouin convaincues de ce crime empêcheront à l'avenir de pareils désordres.» ${ }^{57}$

Une des raisons de la sévérité de l'Édit d'Henri II découle de l'enseignement de l'Église catholique qui stipule que les enfants non baptisés ne connaîtront jamais le bonheur du ciel et n'ont pas droit à une sépulture religieuse, enseignement également dispensé par certaines dénominations protestantes ${ }^{58}$. On retrouve des traces de cette préoccupation dans les documents judiciaires jusqu' au XXe siècle. En 1850, les actes d'accusation portés contre Adèle A. et Bridget B. précisent que les enfants qu'elles avaient fait mourir n'étaient pas baptisés ${ }^{59}$. En 1906, le docteur Brunet reproche à Céleste C. d'avoir accouché seule parce que «faute d'un médecin, l'enfant sera privé de la présence de Dieu durant toute l'éternité.» Pour la même raison, un détective dit à Denise D. que «c'était bien de valeur que son enfant était mort sans

53 François-André Isambert, Recueil des anciennes lois françaises depuis l'an 420 jusqu'à la Révolution de 1789 (Paris, 1827-1829), 13: 471-473.

54 Il s'agit de Françoise Duverger en 1671, Madeleine Gibaut en 1697, toutes deux de Montréal, et Marie-Anne Sigouin de Québec en 1732. 24.

${ }_{55}$ Michel Foucault, Surveiller et punir. Naissance de la prison (Paris, Gallimard, 1975),

56 ANQ, Conseil souverain, Procédure judiciaire criminelle, vol. 3, f. 88-183, 1708, procès de Marie-Barbe Dupont veuve Pinel; vol. 4, f. 134-212, 1732, procès de Marie-Anne Sigouin.

57 Archives des colonies, C11A, vol. 60, Hocquart au ministre, 3 octobre 1733 (microfilm au ANQ).

${ }_{58}$ Ann Jones, op. cit., 50.

59 ANQ, CSP, b. 126 , nos 12 et 38 , janvier 1850. 
avoir été ondoyé.» ${ }^{60}$ La déclaration de Alice A. en 1924, qui affirme avoir baptisé son enfant avec de la salive, reflète le même désir de se conformer aux règles religieuses ${ }^{61}$.

Après le Conquête, l'Édit d'Henri II est remplacé par la loi de Jacques I qui lui ressemble sur plus d'un point. En effet, d'après cette loi, toute femme qui dissimule la naissance d'un enfant bâtard est tenue responsable de son décès et punie de mort, à moins qu'elle puisse produire un témoin attestant que l'enfant est mort-né62. Cette loi, qui est également appliquée dans les colonies américaines, reste en vigueur jusqu'en 1803 en Angleterre et 1812 au Bas-Canada ${ }^{63}$. Pendant la période 1760-1812, trois procès pour infanticide ont lieu dans le district judiciaire de Québec, mais nous n'avons pas retrouvé les sentences de deux d'entre eux. Le troisième se déroule en 1766 et crée une vive émotion populaire. L'accusé avait été chargé de remettre une enfant de deux semaines aux religieuses de l'Hôtel-Dieu, mais il l'abandonne dans la neige, près de l'hôpital, par une nuit de février. Le coupable échappe à la potence grâce au pardon royal, mais il doit affronter la colère populaire. À sa sortie de prison, trois hommes l'attaquent et le rouent de coups $^{64}$.

En résumé, les lois de l'Ancien régime se caractérisaient par une grande sévérité. Lors des procès pour infanticide, aucune circonstance atténuante n'était reconnue aux accusées. Mais les procédures d'appel et de grâce permettaient d'adoucir certaines sentences, après que le juge eût prononcé une condamnation à mort «pour l'exemple». Ce désir de «faire un exemple», était au centre des préoccupations des juges de l'époque. Cette mentalité va évoluer mais on peut en observer, occasionnellement, des résurgences.

\section{2 - La législation moderne}

Dans la deuxième moitié du XVIIIe siècle, des philosophes et des humanistes remettent en question l'ensemble du système pénal. Les réformes qu'ils préconisent visent essentiellement à corriger les criminels, en les punissant par l'emprisonnement au lieu de les mettre à mort, et à prévenir le crime dans la mesure du possible. L'un de ces réformateurs, Cesare Beccaria, célèbre pour ses prises de position contre la peine de mort et la torture, accorde une attention particulière à l'infanticide:

L'infanticide est le résultat presque inévitable de l'affreuse alternative où se trouve une infortunée, qui n'a cédé que par faiblesse,

\footnotetext{
60 ANQ, CS, crim., avril 1906; CSP, IP, 10 mars 1910.

61 ANQ, CS, EC, b. 2347, no 109, 11 mars 1924.

62 La loi de Jacques I est datée de 1623 ou 1624, selon les auteurs qui en parlent. Davies, op. cit., 213; Kellum, op. cit., 371; Malcolmson, op. cit., 196; Wrightson, op. cit., 11; Backhouse, op. cit., 449.

63 Backhouse, op. cit., 454; Jones, op. cit., 45.

64 ANQ, CS, EC, 3 février 1766; CS, crim., février-juin 1766.
} 
ou qui a succombé sous les efforts de la violence. D'un côté, l'infamie, de l'autre la mort d'un être incapable de sentir la perte de la vie: comment ne préférerait-elle pas ce dernier parti, qui la dérobe à la honte, à la misère, elle et son malheureux enfant? [...] On ne peut appeler juste ou nécessaire la punition d'un délit que les lois n'ont pas cherché à prévenir par les meilleurs moyens possibles. ${ }^{65}$

Dans ce texte, Beccaria expose trois opinions au sujet de l'infanticide qui seront souvent débattues au cours des siècles suivants. D'abord il présente les mères infanticides comme des infortunées, victimes de leur faiblesse ou de la violence masculine. Ensuite, il souligne les contraintes sociales qui poussent ces femmes au crime: la crainte de la honte et de la misère. Enfin, il minimise quelque peu l'importance de la mort de l'enfant en présentant celui-ci comme «un être incapable de sentir la perte de la vie». Ces idées sont reprises par le jurisconsulte anglais Jérémie Bentham en 1803:

Quel est le crime? Ce qu'on appelle improprement la mort d'un enfant qui a cessé d'être avant d'avoir connu l'existence, dont l'issue ne peut pas exciter la plus légère inquiétude dans l'imagination la plus craintive, et qui ne peut laisser des regrets qu'à celle même qui, par un sentiment de pudeur et de pitié, a refusé de prolonger des jours commencés sous de malheureux auspices; et quelle est la peine? On inflige un supplice barbare, une mort ignominieuse à une malheureuse mère dont le délit même prouve l'excessive sensibilité, à une femme égarée par le désespoir [...], on la dévoue à l'infamie parce qu'elle a trop redouté la honte. ${ }^{66}$

Pour remédier à ce crime, Bentham suggère de punir plus sévèrement les hommes qui s'attaquent aux femmes et il approuve la fondation de "ces asiles secrets d'accouchement, ces hôpitaux pour les enfants trouvés, qui ont prévenu si souvent les effets sinistres du désespoir, en couvrant des ombres du mystère les suites d'un égarement passager». Un siècle plus tard, on retrouve les idées de Beccaria et de Bentham sous la plume du médecin anglais Charles Mercier:

In comparison with other cases of murder, a minimum of harm is done by it $[\ldots]$ The victim's mind is not sufficiently developed to enable it to suffer from the contemplation of approaching suffering or death. It is incapable of feeling fear or terror. Nor is its consciousness sufficiently developed to enable it to suffer pain in appreciable degree. Its loss leaves no gap in any family circle, deprives no children of their bread-winner or their mother, no human being of a friend, helper or companion. The crime diffuses no sense of insecurity. No one feels a whit less safe because the crime has been committed. ${ }^{67}$

65 Cesare Beccaria, Des délits et des peines (Paris, Flammarion, 1979 [1764]), 140-141.

66 Jérémie Bentham, Traités de législation civile et pénale (Paris, Bossang, 1820 [1803]), 2: 44 et 372 .

67 Charles Mercier, Crime and Insanity (London, William and Norgate, 1911), 212. 
Bref, ces trois auteurs estiment que l'infanticide est un crime moins dangereux que les autres, que les mères coupables sont dignes de pitié plutôt que de blâme, et qu'il vaut mieux essayer de prévenir ce crime plutôt que le punir. Ces idées, qui se répandent à partir du début du XIXe siècle, incitent les gouvernements à prendre des mesures d'aide aux enfants trouvés ${ }^{68}$ et à modifier les pratiques judiciaires.

\subsection{1 - La loi de 1812 et les incertitudes de la médecine}

Au début du XIXe siècle, le code criminel anglais prévoit la peine de mort dans un si grand nombre de cas qu'il a été surnommé le «code sanglant». Paradoxalement, l'excès même de sa sévérité nuit à son efficacité. En effet, bien des fois, des jurés inspirés par des sentiments d'humanité hésitent à rendre un verdict de culpabilité contre une personne accusée de vol, par exemple, en sachant que celle-ci sera condamnée à mort. Ils optent pour un verdict de non-culpabilité et son délit reste impuni. Pour remédier à cet état de chose, les législateurs anglais modifient plusieurs fois et remplacent la sentence de mort par une peine plus légère que les tribunaux n'hésiteront pas à appliquer ${ }^{69}$.

C'est dans ce contexte que la loi de Jacques I est abrogée en Angleterre en 1803, puis au Bas-Canada en $1812^{70}$. Une nouvelle loi stipule que lors des procès pour meurtre d'enfant bâtard, il faudra suivre les mêmes règles que dans les autres procès pour meurtre, c'est-à-dire que l'accusée sera présumée innocente et que le fardeau de la preuve incombera à la poursuite. Il s'agit là d'un changement fondamental, car précédemment l'accusée était présumée coupable et devait prouver ellemême son innocence. La même loi ajoute que même si l'accusée est déclarée non coupable de meurtre, le juge peut la condamner à deux années d'emprisonnement si elle a essayé de cacher cette naissance ${ }^{71}$. Au fil des années, des précisions sont apportées. Ainsi il n'est pas nécessaire de prouver que l'enfant est né mort ou vivant pour déclarer

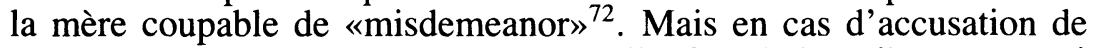
meurtre, il faut prouver que le corps de l'enfant était entièrement sorti du sein de la mère: il n'est pas suffisant qu'il ait respiré au cours de l'accouchement. On émet aussi la possibilité de porter une accusation

68 Au début, cette politique fut critiquée. En 1815, la Gazette de Québec commenta un cas d'infanticide en s'élevant contre «cette institution [le tour de l'Hôtel-Dieu] qui, en même temps qu'elle opère comme une gratification pour les vicieux en les déchargeant du soin de leurs enfants, est inefficace pour empêcher le crime». La Gazette de Québec, 16 février 1815, 3. Cité par JeanMarie Fecteau, Un nouvel ordre des choses: la pauvreté, le crime, l'État au Québec, de la fin du XVIIIe siècle à 1840 (Montréal, VLB éditeur, 1989), 152-153.

69 Arthur Koestler, «Réflexions sur la potence», Albert Camus et Arthur Koestler, Réflexions sur la peine capitale (Calmann-Lévy, 1986), 43; Jean-Marie Fecteau, op . cit., 167; R. Sauer, op. cit., 82.

1812 La loi de Jacques I fut rappelée au Nouveau-Brunswick en 1810, au Bas-Canada en 1812, en Nouvelle-Écosse en 1813, au Haut-Canada en 1826. Voir Backhouse, op cit., 454.

$7_{11}$ Les Statuts provinciaux du Bas-Canada (Québec, Desbarats, 1811 [sic]), 7: 137-139.

72 Jacques Crémazie, Les lois criminelles anglaises arrangées suivant les dispositions introduites dans le code criminel de cette province (Québec, Fréchette, 1842), 82 et 88. 
d'homicide involontaire coupable («manslaughter») lorsque le nouveau-né meurt des suites d'une blessure mortelle infligée "sans malice» ${ }^{73}$. Enfin, l'accusation de suppression de part peut être portée non seulement contre la mère, mais aussi ses complices ${ }^{74}$.

Pendant la période qui va de 1812 à 1891,29 procès ont lieu à la suite du décès d'un nouveau-né, dont 19 comprennent une accusation de meurtre (voir tableau 3). Dans de tels cas, il importe de déterminer

TABLEAU 3

Procès faisant suite à la mort d'un nouveau-né dans le district de Québec

1812-1891

\begin{tabular}{|c|c|c|c|c|c|}
\hline \multicolumn{2}{|l|}{ Accusation } & \multicolumn{2}{|c|}{ Verdict } & \multicolumn{2}{|l|}{ Sentence } \\
\hline Meurtre & $: 19$ & $\begin{array}{l}\text { Non lieu } \\
\text { Non coupable } \\
\text { «Discharged» } \\
\text { «Convicted» }\end{array}$ & $\begin{array}{lr}: & 5 \\
: & 12 \\
: & 1 \\
: & 1\end{array}$ & 6 mois prison & $: 1$ \\
\hline $\begin{array}{l}\text { Suppression } \\
\text { de part : } \\
\text { (concealing } \\
\text { of birth) }\end{array}$ & $: 18$ & $\begin{array}{l}\text { Non lieu } \\
\text { Non coupable }\end{array}$ & $\begin{array}{ll}: & 2 \\
: & 1\end{array}$ & 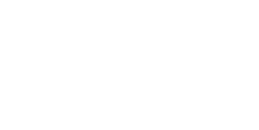 & \\
\hline & & $\begin{array}{l}\text { «ischarged» } \\
\text { Coupable }\end{array}$ & $\begin{array}{l}: 4 \\
: 11\end{array}$ & $\begin{array}{l}2 \text { ans travaux forcés } \\
1 \text { an travaux forcés } \\
1 \text { an prison } \\
6 \text { mois travaux forcés } \\
6 \text { mois prison } \\
4 \text { mois prison } \\
2 \text { mois prison } \\
6 \text { semaines prison }\end{array}$ & $\begin{array}{ll}: & 1 \\
: & 1 \\
: & 2 \\
: & 1 \\
: & 3 \\
: & 1 \\
: & 1 \\
: & 1\end{array}$ \\
\hline «Infanticide» & $: 4$ & $\begin{array}{l}\text { Non lieu } \\
\text { «Discharged» }\end{array}$ & $\begin{array}{ll}: & 2 \\
: & 2\end{array}$ & & \\
\hline $\begin{array}{l}\text { Homicide } \\
\text { involontaire } \\
\text { coupable } \\
\text { (manslaughter) }\end{array}$ & $: \quad 1$ & Non lieu & $: 1$ & & \\
\hline
\end{tabular}

Source: ANQ et Préarch. Voir note 6.

N.B. On aboutit au total de 42 accusations pour 29 procès parce que dans 11 cas, on porte 2 accusations contre une même personne (meurtre et suppression de part) et dans un autre cas, une triple accusation de meutre, suppression de part et «manslaughter».

73 Henri-Elzéar Taschereau, The Criminal Law Consolidation and Amendment Acts (Montréal, 1874), 181-182. En 1931, encore, un juge de Montréal rappela aux jurés qu'il fallait que l'enfant soit entièrement sorti du corps de la mère pour être considéré comme un être humain victime d'un meurtre. Voir Andrée Lévesque, La norme et les déviantes. Des femmes au Québec pendant l' entre-deux-guerres (Montréal, Editions du Remue-ménage, 1989), 116.

74 Raoul Dandurand et Charles Lanctôt, Traité théorique et pratique de droit criminel (Montréal, Périard, 1890), 284. George Wheelock Burbidge, A Digest of the Criminal Law of Canada (Toronto, Carswell and Co., 1890), 209, 228-229. 
si l'enfant est né vivant. Pour le savoir, les médecins procédaient au test de la docimasie pulmonaire, c'est-à-dire qu'ils plongeaient les poumons dans l'eau. S'ils surnagent, on conclut que l'enfant a respiré. Mais la validité de cette méthode est contestée dès $1841^{75}$. Compte tenu des limites de leur science et des exigences de la loi, on comprend que les médecins aient du mal à rendre un diagnostic sûr. Leur incertitude apparaît avec une évidence particulière dans les cas d'Elsie E. et de Fanny F. en 1851. La première, une servante, avait accouché en secret et le cadavre de son enfant fut retrouvé gelé dans un fenil. Les deux médecins qui procèdent à l'autopsie constatent que les poumons flottent dans l'eau, mais ils sont incapables de préciser si l'entrée de l'air dans les poumons a eu lieu avant ou après l'accouchement. L'un de ces médecins rend exactement le même diagnostic dans le cas de Fanny F. qui a accouché dans le bois et dissimulé le corps de l'enfant sous une écorce de bouleau ${ }^{76}$.

Dans d'autres cas, tout en indiquant avec précision les causes de la mort, les médecins accompagnent leur verdict de commentaires qui peuvent être interprétés comme un motif valable pour disculper la mère. En 1875, par exemple, Ursule U., une autre servante, avait accouché seule dans le bois, et on retrouve l'enfant caché sous un tas de feuilles. Les jurés du coroner concluent qu'il était «mort de syncope, suite de l'hémorragie occasionnée par la lacération du cordon ombilical par sa mère», et que celle-ci l'avait volontairement assassiné. Mais le rapport d'autopsie affirme que «le même effet peut être produit par une femme qui accouche debout.» Et la Cour rend un verdict de non-lieu ${ }^{77}$. D'une façon générale, quand la mère de l'enfant est connue, le diagnostic de mort violente est moins fréquent que lorsque le nouveau-né est un inconnu $(37,6 \%$ de cas en regard de $45,8 \%)$. Et en cas d'incertitude, les médecins tentent de faire jouer le doute en faveur de l'accusée. Il en résulte qu'une seule des accusations de meurtre entraîne la condamnation de la mère.

L'accusation de suppression de part (concealing of birth) aboutit plus souvent à une condamnation (11 cas sur 18) car la preuve est plus facile à apporter. Dans certains cas, il ne fait pas de doute que la mort de l'enfant est due à une intervention humaine. Par exemple, en 1844, l'enfant de Harriet $\mathrm{H}$. est retrouvé dans un coffre. Le médecin déclare qu'il a complètement respiré et est mort par suffocation. Deux ans plus tard, l'enfant d'Irène I., une servante, est trouvé dans les latrines, portant des marques de violence sur la tête. En 1882, on découvre l'enfant de Julie J. près de l'Hôtel-Dieu et le rapport d'autopsie attribue la mort «à des actes de violence, les ecchymoses de la tête et du cou en sont la

\footnotetext{
75 Crémazie, op. cit., 82. Bien que contestée, cette méthode continua d'être employée au Québec lors des enquêtes du coroner.

76 ANQ, CS, EC, 12 février $1851 ; 3$ juillet 1851

77 ANQ, CS, EC, 2 juillet 1875.
} 
preuve» ${ }^{78}$. Bien qu'il soit évident, à la lumière des circonstances, que ces enfants ont été tués, le tribunal juge que ces trois femmes ne sont coupables que de suppression de part et les condamne respectivement à 12 mois, 6 mois et 6 semaines de prison. Dans l'ensemble, les sentences sont d'ailleurs très modérées. La peine maximale de deux ans de travaux forcés est imposée une seule fois (tableau 3). Quant à Fanny F. et Ursule U., elles ne reçoivent aucune sentence, bien qu'il soit certain qu'elles ont essayé de cacher la naissance (et la mort) de leur enfant.

Il apparaît à la lecture des enquêtes et des procès que les médecins et les hommes de loi évitent d'accabler les accusées. Non seulement ils acceptent d'emblée le changement de loi de 1812, mais ils s'efforcent même d'en mitiger les effets en imposant des sentences plus faibles que celles prévues. La même attitude de clémence a été constatée en Ontario, en Angleterre et en France, même si dans ces deux pays on a relevé quelques rares exécutions pour infanticide au XIXe siècle ${ }^{79}$. Visiblement le discours des philanthropes qui s'apitoyaient sur le sort des fillesmères avait été entendu, tout au moins par les élites cultivées.

\subsection{2. - Le code criminel de 1892: la négligence criminelle et les façons d'excuser la mère infanticide}

Au cours du XIXe siècle, des médecins ont souvent déclaré en cour que la mort de plusieurs nouveau-nés dépendait du manque total de soins à la naissance. L'article 239 du code criminel, promulgué en 1892, apparaît comme une réponse à cet état de fait:

Est coupable d'un acte criminel toute femme qui, [...] étant enceinte et sur le point d'accoucher, néglige de se procurer l'aide raisonnable pour son accouchement, si par là elle fait un tort permanent à son enfant ou s'il meurt [...] et est passible des peines suivantes: a) Si le but de cette négligence était que l'enfant ne vécût pas, l'emprisonnement à perpétuité; b) Si son but était de cacher le fait qu'elle a eu un enfant, l'emprisonnement pendant 7 ans. ${ }^{80}$

En mettant l'accent sur la négligence commise par la mère, le législateur assimile son geste à un homicide involontaire coupable (manslaughter), crime commis lorsque: «a man, by culpable neglect of a duty imposed upon him, is the cause of the death of another.» ${ }^{81} \mathrm{En}$ outre, comme l'explique l'avocat James Crankshaw, cette nouvelle loi permettrait de punir certains meurtres d'enfant en reconnaissant coupable de «manslaughter», et non de meurtre, «a woman who caused

78 ANQ, CS, crim., b. 2273, no 7, 3 août 1844; CSP, b. 138, février-août 1844; CS, crim., b. 2273, février 1846; CS, EC, b. 2318, no 17, 12 août 1882. 335 .

Backhouse, op. cit.; Behlmer, op. cit., 142; Davies, op. cit., 217-219; Briand, op . cit.,

80 Code criminel du Canada (Ottawa, 1892), articles 219, 239, 240.

81 S. R. Clarke, A Treatise of Criminal Law as Applicable to the Dominion of Canada (Toronto, Carswell, 1872), 252. 
her new born child's death by an act done when her power of selfcontrol was greatly weakened ${ }^{82}$.

Cette allusion à une perte de maîtrise chez la mère marque une étape importante dans l'évolution des lois sur l'infanticide. Depuis le XVIIe siècle, en Angleterre, des jurés avaient évoqué la «frénésie temporaire» et la «manie puerpérale» pour expliquer le geste infanticide. En 1872, on propose d'adoucir la sentence dans les cas de meurtre de nouveau-né par une mère qui a perdu le contrôle d'elle-même ${ }^{83}$. À la même époque, plusieurs auteurs français (avocats, psychiatres, médecins) se penchent également sur cette question. Un traité français de médecine légale résume ainsi une opinion qui se répand à l'époque:

L'infanticide est l'oeuvre d'un instant d'égarement plus encore que l'oeuvre de la perversité [...] S'il arrive quelquefois qu'une mère vienne à violer froidement les lois les plus sacrées de la nature, plus souvent, encore, dans son isolement, dans son désespoir, en proie aux tortures physiques et aux souffrances morales, elle n'a plus la conscience de ses actions; ses sens se troublent, sa raison s'égare: c'est le délire, dit Esquirol, qui conduit ses mains sacrilèges. ${ }^{84}$

À partir de 1892, la loi prévoit donc qu'une femme nouvellement accouchée peut commettre deux types de crime: laisser mourir son enfant faute de soins parce qu'elle est physiquement trop faible pour s'en occuper, ou le tuer de ses propres mains parce qu'elle a perdu la maîtrise de ses actes à la suite de l'accouchement.

Les peines prévues par l'article 239 sont plus lourdes que pour la suppression de part qui garde force de loi (article 240), mais un coup d'oeil jeté sur le tableau 4 révèle qu'elles ne furent jamais appliquées dans toute leur rigueur. Même dans les cas où le meurtre de l'enfant est évident, et sûrement imputable à la mère, le juge n'impose qu'une sentence relativement légère. Prenons l'exemple de Denise D. en 1910: l'autopsie révèle que la mort de son enfant est due à la strangulation et à deux fractures du crâne, mais elle est condamnée à six mois de prison seulement pour suppression de part. En 1929, l'enfant de Louise L. meurt d'asphyxie "par compression du cou avec les mains et l'introduction d'un corps étranger dans la bouche». La mère est accusée d'avoir négligé de se procurer de l'aide pour son accouchement, condamnée à

\footnotetext{
82 James Crankshaw, The Criminal Code of Canada (Montréal, Whiteford et Théoret, 1894), articles 239-240; ibid. (Toronto, Carswell Co, 1915), articles 271-272. cit., 13, 15 .

Behlmer, op. cit., 413; Walker, op. cit., 127, 129; Sauer, op. cit., 83; Wrightson, op.

84 Briand et Chaudé, op. cit., 335. N'allons pas en conclure, cependant, que tous les hommes de loi français témoignaient de la sympathie aux filles-mères infanticides. Au contraire, René Bouton, op . cit., 128 et 194, par exemple, s'indigne de la sensiblerie des juges qui s'apitoyaient sur la fille séduite et rejetaient le blâme sur l'infâme séducteur. C'était également l'opinion de l'Anglais Charles Mercier.
} 
TABLEAU 4

Procès faisant suite à la mort d'un nouveau-né dans le district de Québec

1892-1947

\begin{tabular}{|c|c|c|c|c|c|}
\hline \multicolumn{2}{|l|}{ Accusation } & \multicolumn{2}{|c|}{ Verdict } & \multicolumn{2}{|l|}{ Sentence } \\
\hline Meurtre & $: 5$ & Non coupable & $: 5$ & & \\
\hline Suppression de part & $: 16$ & Coupable & $: 16$ & $\begin{array}{l}15 \text { mois prison } \\
2 \text { à } 6 \text { mois prison } \\
6 \text { à } 17 \text { jours prison } \\
\text { Internement } \\
\text { au Bon-Pasteur } \\
\text { Sentence suspendue }\end{array}$ & $\begin{array}{l}: 1 \\
: 9 \\
: 3 \\
: 2 \\
: 1\end{array}$ \\
\hline Négligence & $: 13$ & $\begin{array}{l}\text { Non lieu } \\
\text { Non coupable } \\
\text { Pas indiqué } \\
\text { Coupable }\end{array}$ & $\begin{array}{ll}: & 1 \\
: & 2 \\
: & 1 \\
: & 9\end{array}$ & $\begin{array}{l}10 \text { à } 23 \text { mois prison } \\
1 \text { à } 5 \text { mois prison } \\
\text { Sentence suspendue }\end{array}$ & $\begin{array}{l}: 3 \\
: 3 \\
: 3\end{array}$ \\
\hline Homicide involontaire & & Non lieu & $: 2$ & 23 mois prison & $: 1$ \\
\hline Coupable & $: 6$ & $\begin{array}{l}\text { Non coupable } \\
\text { Coupable }\end{array}$ & $\begin{array}{ll}: & 2 \\
: & 2\end{array}$ & 2 mois prison & $: 1$ \\
\hline «Infanticide» & $: 1$ & Coupable & $: 1$ & 3 mois prison & $: 1$ \\
\hline
\end{tabular}

Source: ANQ et Préarch., voir note 6.

N.B.: On aboutit au total de 41 accusations pour 32 procès parce que, dans 9 cas, 2 accusations sont portées contre l'accusée.

TABLEAU 5

Procès faisant suite à la mort d'un nouveau-né dans le district de Québec

1948-1969

\begin{tabular}{|c|c|c|c|c|c|}
\hline \multirow{2}{*}{$\begin{array}{l}\text { Accusation } \\
\text { Infanticide }\end{array}$} & \multirow[b]{2}{*}{$: 4$} & \multicolumn{2}{|c|}{ Verdict } & \multicolumn{2}{|l|}{ Sentence } \\
\hline & & Coupable & $: 4$ & $\begin{array}{l}12 \text { mois prison } \\
1 \text { à } 4 \text { mois prison } \\
\text { Temps déjà fait en } \\
\text { prison (22 jours) }\end{array}$ & $\begin{array}{l}: 1 \\
: 2 \\
: 1\end{array}$ \\
\hline Supression de part & $: 4$ & $\begin{array}{l}\text { Coupable } \\
\text { Plainte retirée }\end{array}$ & $\begin{array}{ll}: & 3 \\
: & 1\end{array}$ & $\begin{array}{l}\text { Sentence suspendue } \\
1 \text { mois prison } \\
\text { Temps fait en prison } \\
\text { ( } 73 \text { jours) }\end{array}$ & $\begin{array}{l}: 1 \\
: 1 \\
: 1\end{array}$ \\
\hline
\end{tabular}

Source: Préarch., voir note 6.

N.B. On arrive au total de 8 accusations pour 7 procès parce que dans un cas on porte une double accusation pour infanticide et suppression de part. 
un mois de prison et libérée le lendemain ${ }^{85}$. En 1933, Mathilde M., une servante de 22 ans, admet qu'elle a mis au monde un enfant vivant, mais «comme il pleurait, elle l'avait d'abord étouffé et ensuite fini de le tuer [...] en le dardant au coeur avec des ciseaux» ${ }^{86}$. Elle se reconnaît coupable d'homicide involontaire et est condamnée à 23 mois de prison. Bref, l'habitude prise par les juges, avant 1892, d'imposer des sentences de moins de deux ans de prison, se maintient sans discontinuer. Les dossiers des procès du XXe siècle, plus étoffés et mieux conservés que ceux des époques précédentes, renferment l'explication de cette attitude.

La correspondance échangée entre les officiers de justice chargés d'instruire un procès, les lettres d'intercession des curés, les notes prises par les juges et les interrogatoires lors des enquêtes, tout cela révèle les réactions face au phénomène de l'infanticide et les circonstances susceptibles de susciter la sympathie à l'endroit des accusées.

L'argument de l'honneur, celui de l'accusée et de sa famille, conserve encore au XXe siècle toute sa pertinence. En 1907, pour excuser une vieille dame qui avait aidé sa servante à se débarrasser du cadavre de son enfant, un officier de police déclare: «Cette femme n'a pas agi avec intention de commettre un crime; c'était plutôt pour sauver la réputation de la fille.» En 1928, un curé emploie un argument semblable pour aider un de ses paroissiens accusé d'avoir fait disparaître le corps de l'enfant de sa fille: «Il a certainement jeté cet enfant sans réfléchir, uniquement soucieux d'éviter un scandale à ses grands garçons.» ${ }^{87}$

L'ignorance est une autre excuse évoquée de façon toute paternaliste par les curés. Nestor N. est présenté par son curé comme un «minus habens» qui avait sans doute agi sur le conseil de sa femme. Un autre curé intercède en faveur d'une paroissienne, «une malheureuse jeune fille» qui avait jeté son enfant dans un cabinet de toilette: «Vu son jeune âge, elle a dû agir avec plus d'ignorance que de malice.» Dans les deux cas, les prêtres estiment que le chagrin et la honte ressentis par les familles constituent une punition suffisante ${ }^{88}$. Les magistrats se montrent sensibles à ce genre d'argument. En 1935, Viviane V. qui a accouché seule dans une maison de campagne et caché le cadavre de l'enfant sous un tas de fumier, est condamnée à trois mois de prison seulement parce qu'elle a paru au juge «très peu intelligente, n'ayant reçu que peu ou pas d'éducation». Nathalie N. qui agit de façon iden-

85 ANQ, CSP, IP, b. 600, no 3, 10 mars 1910; CSP, b. 213, no 59, 23 mars 1910; CS, EC, b. 2350, no 290, 14 janvier 1929; CSP, b. 257, no 1510, 15 février 1929; Écrou de la prison de Québec, 1922-1932.

86 ANQ, CSP, EC, b. 2, no 1194, 31 août 1933; CSP, b. 345, no 9251, 18 novembre 1933.

87 ANQ, DPG, no 1395, mars-avril 1907; CSP, b. 245, no 448, mai-juin 1928.

88 ANQ, CSP, b. 245, no 448, mai-juin 1928; DPG, no 3576, septembre 1907. 
tique en 1938, reçoit une sentence de quinze jours de prison seulement «à cause de son ignorance» ${ }^{89}$.

Les hommes de loi tiennent compte aussi de la perte de maîtrise de soi de la femme après l'accouchement. C'est le procès de Peggy $P$. en 1922, qui révèle le mieux les efforts d'un juge qui emploie tous les moyens pour disculper une fille. L'accusée, une servante de 23 ans, a accouché en secret et le médecin légiste affirme que l'enfant avait respiré et qu'il était mort d'une fracture du crâne. Lors de l'instruction préliminaire, le juge interroge le médecin:

Q: L'enfant en tombant par terre, pouvait-il se causer cette fracture?

R: Non. Pas une fracture aussi marquée.

$\mathrm{Q}$ : Est-ce que, dans l'état où elle se trouve, une femme, et surtout une jeune fille, dans cette circonstance-là, en se levant, a pu laisser l'enfant sur le plancher, et, qu'il aurait pu se causer cette blessure?

R: Le fait qu'une femme accouche debout et que l'enfant tombe, ne cause jamais les blessures que j'ai trouvées.

$\mathrm{Q}$ : Est-ce qu'une femme, dans les douleurs où elle se trouve, et accouchant seule, ne pourrait pas blesser de cette manière-là, en voulant aider à la sortie de l'enfant?

$R$ : Ce n'est pas du tout l'endroit où j'ai trouvé les marques que les mains auraient fait.

Q: Est-ce qu'une femme, dans l'état malade ou souffrant comme cela, sans le faire exprès, pourrait prendre un enfant et lui serrer la tête ou la gorge de cette manière-là, sans se rendre compte de ce qu'elle fait, dans l'état où était cette pauvre jeune fille?

$\mathrm{R}$ : C'est difficile à répondre; si la femme était complètement aliénée, je comprends.

Q: Mais une femme dans cet état-là peut facilement perdre la raison momentanément?

R: Pas facilement, non.

Q: Remarquez bien que cette jeune fille-là était dans une position bien difficile; elle voulait cacher la naissance de son enfant à ses maîtres; alors, ne pourrait-elle pas être dans un état d'esprit tel qu'elle pût ne pas savoir ce qu'elle faisait et, poignant cet enfant-là dans ses mains, a pu lui faire les blessures que vous avez dites?

R: Non. C'est pas possible, faites rien qu'avec les mains.

$\mathrm{Q}$ : Ni avec ses ongles, elle n'a pas pu lui faire ces blessures, ces marques en zig-zag?

R: Non. Par pour moi.

Q: N'est-il pas vrai qu'une femme, dans l'état où se trouvait cette jeune fille, a une force considérable dans les mains?

$\mathrm{R}$ : Je ne vois pas pourquoi elle serait plus forte qu'auparavant. ${ }^{90}$

89 ANQ, CSP, EC, b. 12, no 2098, 13 mai 1935; CSP, b. 374, no 11731, 7 juin 1935; CSP, b. 426, no 15943,27 mai 1938.

90 ANQ, CSP, IP, b. 106, 27 février 1922. 
À travers ce dialogue, on sent le désir du juge d'obtenir une réponse qui lui permettrait de lever l'accusation qui pèse sur la fille. Il essaie de faire dire au médecin que l'enfant a pu se tuer accidentellement, ou que la mère a pu le blesser involontairement. Ses allusions aux souffrances de l'accouchement, à la solitude de la jeune fille montrent bien où va sa sympathie. Les jurés manifestent les mêmes sentiments lorsqu'ils recommandent l'accusée à la clémence de la cour ${ }^{91}$.

L'attitude de ce juge de même que les interventions des curés en faveur de leurs paroissiens peuvent donner l'impression que les personnes accusées d'infanticide jouissaient d'une sympathie universelle. Mais ce n'était pas toujours le cas. Des fragments de la correspondance du procureur général révèlent plutôt que les sentiments du public se partageaient entre l'indignation et la sympathie. Décrivant la situation d'une jeune fille de sa paroisse accusée de ce crime, en 1907, un curé écrit: «Le scandale a été immense (...) L'indignation publique [est] soulevée contre elle.» Deux ans plus tard, un nouveau-né est trouvé, exposé au froid, à l'entrée d'un couvent. Deux avocats commentent l'événement: «Ce n'est pas la première fois que des choses de ce genre se passent ici et le public réclame un châtiment qui serve d'exemple salutaire.» Dans le cas de Felicity F., enfin, cette servante accusée d'avoir fait disparaître son enfant nouveau-né, le chef de police écrit en 1907: «Je comprends que le peuple est excité dans le moment, mais il y a beaucoup de sympathie, sachant que la personne accusée du crime est une pauvre jeune fille âgée que de 19 ans, obligée de gagner sa vie, que de plus elle me déclare que le père de l'enfant est l'homme pour lequel elle travaillait. $\gg^{92}$ Cette jeune fille fut condamnée à deux ans de pénitencier et l'officier de police affirma que «le peuple est satisfait de la Couronne.» Avec si peu d'exemples, il est cependant difficile d'affirmer que l'opinion populaire est plus sévère que le juge.

Pour la période de l'entre-deux-guerres, certains indices portent à croire que l'opinion publique évolue dans le sens d'une sympathie croissante à l'égard des jeunes filles, accompagnée de critiques contre les institutions religieuses et la société. En 1933, par exemple, Simone $\mathrm{S}$. accouche seule dans un hangar à bois par une journée de décembre, et abandonne son enfant qui meurt de froid. La rumeur se répand, entretenue par certains journaux, qu'on lui a refusé l'entrée à l'Hôpital de la Miséricorde, et on s'apitoie sur son sort ${ }^{93}$. À la même époque, à Montréal, les journaux qui rapportent des cas d'abandon d'enfants font éga-

\footnotetext{
91 ANQ, Cour Sup. crim., b. 2303, avril 1909; b. 2305, octobre 1920; CSP, b. 68, octobre

92 ANQ, DPG, no 3576, septembre 1907; no 1743-09; no 1395, avril 1907

93 AMGBP, Victorin Germain, "Cancans et commérages», Chronique de la Crèche, 18 janvier 1934. Pour mettre fin à ces rumeurs, l'abbé Germain alla interroger la jeune fille qui lui dit qu'elle n'avait pas voulu aller à la Miséricorde parce qu'elle avait entendu dire que les filles y étaient maltraitées.
} 1927. 
lement preuve de sympathie envers la mère et réservent leurs critiques pour la société qui permet de tels drames ${ }^{94}$.

L'article 239 du code criminel marque un regain de sévérité par rapport à la loi de 1812 en prévoyant des peines plus sévères. Malgré cela, les juges ne se départissent pas de l'attitude de clémence qu'ils ont adoptée au XIXe siècle, et pendant toute la première moitié du XXe, ils n'imposent jamais les nouvelles peines prévues.

\subsection{3 - La loi de 1948: le crime d' «infanticide» et ses causes physiologiques}

Bien que non contenu dans le texte même de la loi de 1892, l'affaiblissement des facultés mentales de la mère infanticide est régulièrement évoqué par les juristes dans la première moitié du XXe siècle. En 1948, cette explication sert de base à une loi qui donne une nouvelle définition du crime d'infanticide:

Une femme qui, par un acte ou omission volontaire, cause la mort de son enfant nouveau-né, est réputée ne pas avoir commis un meurtre ou un homicide involontaire si, au moment de l'acte ou omission, elle ne s'était pas complètement remise de l'effet d'avoir donné naissance à cet enfant et si, de ce fait, son esprit était alors déséquilibré, mais elle est réputée avoir commis un acte criminel, à savoir: un infanticide. ${ }^{95}$

Quelques années plus tard, le code criminel précise que ce déséquilibre peut faire suite à la lactation consécutive à la naissance de l'enfant ${ }^{96}$. La peine prévue est de trois à cinq ans de prison.

Les juges de la région de Québec avaient déjà l'habitude, on l'a vu, d'accueillir favorablement toutes les explications qui permettaient de diminuer le degré de responsabilité des jeunes mères infanticides et d'adoucir les sentences. À partir de 1948, on attribue leur perte de contrôle à des raisons d'ordre physiologique. En 1954, par exemple, une jeune fille est exonérée de tout blâme «vu son état de santé antérieur». En 1956 et 1957, deux autres sont jugées non criminellement responsables de la mort de leur enfant, la première «vu son état d'anémie causé par hémorragie post-partum», la seconde, «vu son état de confusion dû à une rétention placentaire ${ }^{97}$. Au besoin, le coroner oriente ses questions de manière à mettre en évidence l'état mental d'origine pathologique de la jeune femme: «Est-ce qu'elle était dans un état d'excitation? Est-ce qu'elle avait l'air désemparé?» Et le témoin de répondre docilement dans le sens qu'on lui indique: «Elle était dans un état d'ex-

\footnotetext{
94 Andrée Lévesque, op. cit., 114-117.

95 Statuts du Canada (1948), 11-12 Geo. VI, 1: 292.

96 Irénée Lagarde, Nouveau code criminel annoté (Montréal, Wilson et Lafleur, 1957), articles 204 et 208

97 Préarch., CSP, EC, b. 1078, no 1149, 12 mars 1954; b. 1623, no 15529, 25 novembre 1956; b. 1623, no 15732,4 mars 1957.
} 
citation, de folie, elle avait les yeux hagards [...] Elle était désemparée, elle a dit: J'étais comme une folle. ${ }^{98}$

Cette nouvelle insistance sur l'état pathologique des mères infanticides n'écarte pas cependant les autres arguments que les coroners et les juges prennent depuis longtemps en considération, soit l'ignorance, la peur et la solitude. Lors d'une enquête, un coroner reproche même à l'entourage de la jeune fille de ne pas avoir mieux veillé sur elle: «C'est le gros bobo, la petite fille a peur et se ferme, elle est toujours seule, là, c'est ceux qui l'entourent qui pouvaient s'apercevoir de ça.» ${ }^{99}$ Une petite fille isolée, apeurée, irresponsable. Telle est l'image que les hommes de loi se font de ces filles-mères et qui suscite leur indulgence.

\section{CONCLUSION}

L'étude de l'infanticide sur une période de trois siècles révèle des traits de permanence frappants dans l'attitude des personnes qui posent ce geste. La grande majorité d'entre elles sont des filles-mères très jeunes, d'un milieu social fort modeste et qui ont agi dans la solitude. Leur justification est toujours la même: elles ont eu peur du déshonneur, de la misère, et ont cédé à un moment d'affolement. Ce drame qui se déroule de la même façon au milieu du XXe siècle qu'au début du XVIIIe affecte cependant un nombre toujours moins grand de filles à mesure que se développent d'autres moyens d'éviter les grossesses non désirées.

À côté de ces aspects permanents, les mesures légales concernant l'infanticide évoluent constamment. Un changement fondamental se produit au début du XIXe siècle avec le passage d'un droit d'Ancien régime punitif à un droit criminel moderne. La fille soupçonnée d'infanticide qui était jadis présumée coupable bénéficie dorénavant des mêmes droits que les autres accusés. Par la suite, les changements législatifs vont tous dans le sens d'une responsabilité diminuée de la fillemère jusqu'à en faire un cas pathologique.

À ces lois plus douces correspond la clémence des tribunaux qui est déjà acquise au début du XIXe siècle et ne se dément jamais par la suite. L'attitude des hommes de loi contient maintes manifestations de paternalisme. «Cette pauvre jeune fille», dit un juge. «Cette petite fille», dit un coroner. Ils la prennent en pitié et recourent à tous les arguments possibles pour l'acquitter ou du moins alléger la sentence. La sympathie du public est moins évidente: les quelques indices que nous avons relevés font voir des réactions fort diverses.

Les ouvrages qui portent sur l'infanticide au Canada anglais, en Angleterre et en France révèlent les mêmes traits: situation précaire des

98 Préarch., CSP, EC, b. 1623, no 15529, 25 novembre 1956.

99 Préarch., CSP, b. 717, no 45095, mars 1952; EC, b. 738, no 4301. 
filles-mères, changements dans la loi et clémence des juges. Au terme de son article sur l'infanticide en Ontario au XIXe siècle, Clémence Backhouse souligne le fait qu'au Québec, l'Église contrôlait plus sévèrement les méthodes contraceptives en plus de diriger un réseau bien organisé d'hospices pour les enfants trouvés, et elle émet l'hypothèse que ces particularités de la société ont pu susciter à l'égard de l'infanticide des attitudes différentes reflétées par la loi ${ }^{100}$. Nous avons constaté qu'il n'en est rien: dans les deux provinces, les lois concernant l'infanticide sont les mêmes et appliquées de façon semblable. À notre avis, ces ressemblances découlent du fait que toutes ces sociétés participent à la même civilisation qui impose des normes de comportement sexuel différentes pour les hommes et les femmes. Aussi longtemps qu'une société tolère la liberté sexuelle des hommes, des grossesses hors mariages en résultent fatalement; et dans la mesure où ces naissances illégitimes constituent un déshonneur pour les jeunes filles et leur famille, il est inévitable que certaines personnes recourent à des moyens extrêmes pour en garder le secret. L'infanticide apparaît donc comme une conséquence inéluctable du double standard sexuel qui a toujours existé dans les sociétés patriarcales. Quant à la clémence des juges, elle s'explique par le fait que les accusées n'étaient pas considérées comme des criminelles dangereuses en raison de leur jeunesse et d'antécédents irréprochables. Bentham et Mercier n'avaient-ils pas souligné que personne dans la société ne se sentait menacé par un crime comme l'infanticide?

Pour les personnes sensibilisées aux problèmes sociaux d'aujourd'hui, il est parfois tentant de chercher dans le passé des précurseurs des revendications actuelles, notamment en présentant les femmes infanticides comme des révolutionnaires, pleines de courage et de ressources. Mais ce n'est pas l'impression que nous avons retirée de l'analyse des actes judiciaires. Les filles-mères nous apparaissent plutôt comme entièrement dépassées par les événements, montrant un courage qui s'apparente plutôt à l'énergie du désespoir. Plus débrouillardes étaient celles qui recouraient aux oeuvres charitables pour préserver leur réputation tout en procurant à leur enfant des chances raisonnables de survie. Mais les plus énergiques de toutes étaient certainement celles qui décidaient de garder leur enfant et d'affronter les tribunaux pour obtenir une aide pécuniaire du père putatif ${ }^{101}$. Dans la société patriarcale traditionnelle, il fallait plus de détermination pour assumer la condition de fille-mère que pour y échapper en supprimant l'enfant.

100 Backhouse, op. cit., 476.

101 Voir l'étude de Ward, op. cit., et notre article «Filles-mères, famille et société sous le Régime français», Histoire sociale/Social History, 21,41 (mai 1988): 39-69, et «Les filles-mères devant les tribunaux, 1850-1969", projet de communication accepté pour le Congrès de l'Institut d'histoire de l'Amérique française, octobre 1990. 